BROWN-HET-1019

PAR-LPTHE 95-48

December 1995

\title{
COLLECTIVE FIELD THEORY OF THE MATRIX-VECTOR MODELS
}

\author{
by \\ Jean AVAN円 \\ and \\ Antal JEVICKI \\ Department of Physics \\ Brown University \\ Providence, Rhode Island 02912 USA
}

\begin{abstract}
We construct collective field theories associated with one-matrix plus $r$ vector models. Such field theories describe the continuum limit of spin Calogero Moser models. The invariant collective fields consist of a scalar density coupled to a set of fields in the adjoint representation of $U(r)$. Hermiticity conditions for the general quadratic Hamiltonians lead to a new type of extended non-linear algebra of differential operators acting on the Jacobian. It includes both Virasoro and $S U(r)$ (included in $\operatorname{sl}(r, \mathbf{C}) \times s l(r, \mathbf{C})$ ) current algebras. A systematic construction of exact eigenstates for the coupled field theory is given and exemplified.
\end{abstract}

\footnotetext{
${ }^{1}$ Permanent address: LPTHE Paris 6, CNRS URA 280; Box 126, 4 Place Jussieu, F-75252, Paris Cedex 05.
} 


\section{Introduction}

Numerous studies have been devoted to the problem of collective field theory of matrix models. The one-matrix Hamiltonian problem is by now well understood in this approach [1]. The underlying $w_{\infty}$ algebra of observables plays the role of a spectrum-generating symmetry [2]. The connection to quantum Calogero Moser models was also extensively studied [3, 4, 5, 6]. Recently various generalizations, related to multimatrix models [7, 8, 9, 10] have been considered. The relevant space of observables was shown to be the space of marked loops acted upon by splitting and joining operators. The difficulty of manipulating non-local operators on such a huge space and the complexity of the relevant algebraic structures has lead to introducing simplifications of this approach [7, 8].

We investigate here a set of theories intermediate between the one- and multi-matrix models. We are going to construct the collective field theory for a dynamical system defined on a phase space comprising a hermitian matrix variable $m_{i j}, i, j=1 \cdots N$ or equivalently its unitary exponential $M \equiv \exp i m$ with a conjugate momentum field $p_{i j}$, plus a set of $r$ complex vector variables $\left\{x_{i}^{a}, a=1 \cdots r ; i=1 \cdots N\right\}$ and their conjugate momenta $y_{i}^{a}=\frac{\partial}{\partial x_{i}^{a}}$. The group $S U(N)$ has a natural hamiltonian action on this phase space, and we may therefore define collective models with a Hilbert space consisting of functions of the $S U(N)$-invariant variables $\left\{\operatorname{Tr} M^{n} \equiv \phi_{n}^{0}, \operatorname{Tr}\left(\bar{x}^{a} M^{n} x^{b}\right) \equiv \psi_{n}^{a b}, a, b=1 \cdots r\right\}$. The variables $\psi^{a b}$ now carry two $U(r)$ indices, and it will be seen that the field theories which we develop here will describe a consistent interaction of a collective boson $\phi$ with $U(r)$ current algebra degrees of freedom. These models have a number of interesting applications. First of all and most obviously, they are toy models for interacting quarks and open strings [11, 12, 13, 14, 15]. Then, as we will comment, suitable restrictions of the Hilbert space of wave functions for these collective field theories could ultimately provide us with a consistent reduction of any matrix model.

Finally, the most relevant feature of this model, from our present point of view, is that, by Hamiltonian reduction the free matrix Laplacian $H=$

$\sum \frac{\partial^{2}}{\partial m_{i j} \partial m_{j i}}$ acting on the matrix-vector configuration space induces the wellknown spin (or Euler) Calogero-Moser models [16, 17] and the related HaldaneShastry models [18, 19]. Many results were recently obtained on these various 
models, in particular their exact Yangian symmetry [20, 21, 22] and the construction of commuting quantum Hamiltonians by the $R$ matrix method [23] for the Euler Calogero Moser case.

This connection to spin Calogero-Moser models needs to be made more precise. The phase space here contains $r$ complex vector fields and their canonically conjugate (but not hermitean conjugate) momenta. The action of the group $S U(N)$ on the vector fields leads to a contribution to the moment map taking the form, after quantization, of a particular representation of $S U(N)$ :

$$
F_{i j}=\sum_{a=1}^{r} x_{a}^{i} \frac{\partial}{\partial x_{a}^{j}}-\sum_{b=1}^{r} \bar{x}_{b}^{j} \frac{\partial}{\partial \bar{x}_{b}^{j}}
$$

These operators, whichever way they are represented, always define the "spin" interaction of the quantum Euler Calogero Moser models $F_{i j} F_{j i}$ [23]. In this representation the diagonal generators $F_{i i}$ annihilate all collective variables $\phi_{n}^{0}, \phi_{m}^{a b}$, thereby realizing on the reduced $S U(N)$-invariant Hilbert space of wave functions the "zero-weight condition" necessary for integrability of any quantum spin Calogero-Moser model [23]. The fact that it is based on two conjugate vector representations of $S U(N)$ indicates however a closer connection with the integrable $N \otimes \bar{N}$ Calogero-Moser quantum system constructed in [23].

The standard spin Calogero Moser Hamiltonian [16] follows instead from the Hamiltonian reduction of a free theory on a phase space containing one set of $r$ complex vectors together with their canonical and hermitian conjugate momenta. These vectors ought therefore to be parametrized as $a_{k}^{b}=x_{k}^{b}+$ $i p_{k}^{b} ; a_{k}^{b \dagger}=x_{k}^{b}-i p_{k}^{b}$ and the group $S U(N)$ acts on the vectors $a_{k}^{b}, a_{k}^{b \dagger}$, with a contribution to the moment map taking the form of another, oscillator-like, representation of $S U(N)$ :

$$
F_{i j}=\sum_{b=1}^{r} a_{i}^{b \dagger} a_{j}^{b}
$$

The resulting field-theoretic algebraic structures of these models are however identical.

Based upon these introductory remarks our investigations run as follows. In a first part we determine the set of equations obeyed by the Jacobian of the change of variables from the original configuration space $\left\{M, x_{a}^{i}\right\}$ to the 
adjoint-invariant configuration space $\left\{\phi_{n}^{0}, \phi_{m}^{a b}\right\}$. The differential operators acting on $J$ close a non-linear algebra, which we shall obtain explicitly. From the structure of the discrete Hamiltonian we also derive the expected form of the non-hermitian continuum Hamiltonian

In a second part we examine the spectrum of the Hamiltonian corresponding to the reduction of the free matrix Laplacian. We indicate how the structure of the reduced Hamiltonian allows a systematic and exhaustive computation of its eigenvectors and eigenvalues inside the Hilbert space of polynomials in $\left\{\phi_{n}^{0}, \phi_{m}^{a b}\right\}$, and describe fully the diagonalizing procedure. We explicitly apply this procedure to obtain some of the simplest eigenstate with low-lying eigenvalues. We justify the form of both eigenstates and eigenvalues from purely group-theoretical arguments connecting the representation theories of $S U(N)$ and of permutation groups $S_{p}$.

Finally we give a derivation of the hermitian collective field theory at the classical level for the spin Calogero Moser model. The interaction between the scalar and spin-type fields is brought in by the Poisson structure. This derivation also provides us with a semi-classical limit of matrix-vector collective theories, without the technical complications due to the ordering problems and the conjugation by the Jacobian required to get the exact quantum hermitian Hamiltonian. The basic algebraic structure of the Hamiltonian is thus made more transparent.

\section{The Collective Field Theory for the Matrix- Vector Model}

We apply to our configuration space the general formalism defined in [1]. The central issue is the rewriting of an original hermitian Hamiltonian written in terms of variables $(M)_{i j}, \bar{x}_{i}, x_{j}$ as an operator in terms of collective variables

$$
\phi_{n}=\operatorname{Tr}\left(M^{n}\right) ; \psi_{n}^{a b}=\bar{x}^{a} \cdot M^{n} \cdot x^{b}
$$

It is appropriate to indicate here in what sense this series of models may be seen as consistent truncations of a two-matrix problem. It is known (see for instance [10]) that the full two-matrix problem leads to an intricate type of observable space known as loop space with loops parametrized by words 
of arbitrary finite length:

$$
\operatorname{Tr}\left(U_{1}^{n_{1}} U_{2}^{n_{2}} U_{1}^{n_{1}^{\prime}} U_{2}^{n_{2}^{\prime}} U_{1}^{n_{1}^{\prime \prime}} \cdots\right)
$$

This space can be reduced to polynomials in the variables $\phi_{n}=\operatorname{Tr}\left(U_{1}^{n}\right)$ and $\psi_{n}^{a b}=\operatorname{Tr}\left(\bar{x}_{b} x_{a} U_{1}\right)$ by representing $U_{2}$ as $\left(U_{2}\right)^{i j} \equiv \sum_{a=1}^{r} \bar{x}_{a}^{i} x_{a}^{j}+\delta^{i j}$ for some finite $r$. Not every polynomial in this reduced set of variables follows from the reduction of observables of the original multi-matrixmodel. There is a natural action of $U(r)$ on the variables $x, \bar{x}$ viewed as $N r$-component vectors, and $U_{2}$ is invariant under $U(r)$. It easily follows that the polynomials $P\left(\phi_{n}^{0}, \phi_{m}^{a b}\right)$ invariant under the adjoint action of $U\left(r_{2}\right) \times \cdots U\left(r_{p}\right)$ on $\psi_{m}^{a b}$ are the observables of the original multimatrix model.

To derive the collective field theory for the matrix-vector case, we follow the familiar procedure [1]. This essentially requires, beyond an obvious chain rule for derivatives, the introduction of the Jacobian $J$ associated with the change of variables, in order to define the new measure with respect to which the collective Hamiltonian is hermitian. Differential equations obeyed by $J$ then follow and it is in this context that a closed operator algebra arises as Frobenius-Schur conditions on the compatibility of the differential operators acting upon $J$.

Starting from the Hamiltonian, assumed to be at most quadratic in the derivatives $\frac{\partial}{\partial m_{i j}}$ and $\frac{\partial}{\partial x_{i}^{a}}, \frac{\partial}{\partial \bar{x}_{i}^{b}}$, with the most general $U(N)$ invariant form

$$
\begin{aligned}
H & =\sum_{i, j}^{N} \frac{1}{2} m_{M} \frac{\partial^{2}}{\partial m_{i j} \partial m_{j i}}+\sum_{a=1}^{r} \sum_{i=1}^{N} \frac{1}{2} m_{a} \frac{\partial}{\partial \bar{x}_{a}^{i}} \frac{\partial}{\partial x_{a}^{i}} \\
& + \text { potential terms }
\end{aligned}
$$

one applies it to a wave function $\Psi\left(\phi_{n}, \psi_{m}^{a b}\right)$.

For the Hamiltonian given by the matrix Laplacian we obtain (setting $m_{M}=1$ ):

$$
\begin{aligned}
H_{1} & =\frac{1}{2} \sum_{n, m} n m \phi_{n+m} \frac{d^{2}}{d \phi_{n} d \phi_{m}}+\sum_{n, m, a b} n m \psi_{n+m}^{a b} \frac{d^{2}}{d \phi_{n} d \psi_{m}^{a b}} \\
& +\frac{1}{2} \sum_{n, m ; a b, c d} \sum_{u=0}^{n+m} F(n, m, u) \psi_{n+m-u}^{a d} \psi_{u}^{c b} \frac{d^{2}}{d \psi_{n}^{a b} d \psi_{m}^{c d}} \\
& +\frac{1}{2} \sum_{n} n \sum_{u=0}^{n} \phi_{n-u} \phi_{u} \frac{d}{d \phi_{n}}+\sum_{n} \sum_{u=0}^{n}(n-u) \phi_{u} \psi_{n-u}^{a b} \frac{d}{d \psi_{n}^{a b}}
\end{aligned}
$$


with

$$
\begin{aligned}
& F(n, m, u)=u \text { for } 0 \leq u \leq \inf (n, m) \\
& F(n, m, u)=\inf (n, m) \text { for } \inf (n, m) \leq u \leq \sup (n, m) \\
& F(n, m, u)=n+m-u \text { for } \sup (n, m) \leq u \leq n+m
\end{aligned}
$$

while for the vector Laplacian we get:

$$
H_{2}=\sum_{a=1}^{r} \frac{m_{a}}{2}\left\{\sum_{m, n, c d} \psi_{m+n}^{c d} \frac{d^{2}}{d \psi_{m}^{c a} d \psi_{n}^{a d}}+\sum_{m} \phi_{m} \frac{d}{d \psi_{m}^{a a}}\right\}
$$

In (6) $F(n, m, u)$ is in fact a summation kernel and the corresponding term in (6) can be rewritten as

$$
\begin{aligned}
& \sum_{u=0}^{n+m} F(n, m, u)=\sum_{u=0}^{\inf (n, m)} u \cdot+\sum_{\sup (n, m)}^{\sup (n, m)} \inf (n, m) . \\
& +\sum_{\sup (n, m)}^{n+m}(n+m-u) .
\end{aligned}
$$

where the sums are understood algebraically, i.e. $\sum_{a}^{b}=-\sum_{b}^{a}$ if $b \leq a$.

Let us now recall the basic proposition of collective field theory [1]:

Given a Hamiltonian $H$ of the form:

$$
H=\sum_{k}\left\{\sum_{k^{\prime}} \Omega_{k k^{\prime}} \frac{d}{d \phi_{k^{\prime}}}+\omega_{k}\right\} \frac{d}{d \phi_{k}}+V\left(\phi_{k}\right)
$$

the Jacobian which makes $H$ hermitian by conjugation is to obey a set of equations:

$$
\sum_{k^{\prime}}\left\{\Omega_{k k^{\prime}} \frac{d}{d \phi_{k^{\prime}}}+\omega_{k}\right\}^{\dagger} J=0
$$

where $(\dagger)$ denotes the hermitian conjugation for differential operators. The hermitian Hamiltonian then takes the form

$$
\begin{aligned}
H^{h} & =\sum_{k, k^{\prime}} \frac{d}{d \phi_{k}} \Omega_{k k^{\prime}} \frac{d}{d \phi_{k^{\prime}}}-\frac{1}{2} \frac{d^{2} \Omega_{k k^{\prime}}}{d \phi_{k} d \phi_{k^{\prime}}}+V\left(\phi_{k}\right)-\frac{1}{4} \sum_{k} \frac{\partial \omega_{k}}{\partial \phi_{k}} \\
& +\frac{1}{4} \sum_{i, j}\left\{\omega_{i}+\sum_{k} \frac{\partial \Omega_{i k}}{\partial \phi_{k}}\right\}\left(\Omega^{-1}\right)_{i j}\left\{\omega_{j}+\sum_{k} \frac{\partial \Omega_{i k}}{\partial \phi_{k}}\right\}
\end{aligned}
$$


In order for this scheme to be applicable, the set of differential operators (10) must close an algebra or at least generate a closed algebra of constraints under successive commutations. Examples of this construction are the onematrix case realizing a Virasoro algebra [1, [], the general loop space algebra [9, 10] and the Kac-Moody algebra considered in [24]. In this last case the corresponding collective theories were constructed by postulating a Kac-Moody type algebra of constraints and inverting the canonical procedure, thereby going from (10) to (9) and (11).

In order to get the algebra of differential operators annihilating the Jacobian, we write $H$ as:

$$
\begin{aligned}
H & =\frac{1}{2} m_{M} \sum_{n} n O_{n}^{0} \frac{d}{d \phi_{n}}+\frac{1}{2} m_{M} \sum_{n, a b} n\left\{\tilde{O}_{n}^{a b}-\sum_{u=0}^{n-1}\left(1-\frac{u}{n}\right) O_{n, u}^{a b}\right\} \frac{d}{d \psi_{n}^{a b}} \\
& +\frac{1}{2} \sum_{n, a, b}\left\{m_{a} J_{n}^{a b}+m_{b} \bar{J}_{n}^{a b}\right\} \frac{d}{d \psi_{n}^{a b}}
\end{aligned}
$$

where one defines:

$$
\begin{gathered}
O_{n}^{0}=\sum_{q} q \phi_{q+n} \frac{d}{d \phi_{q}}+\sum_{q, c d} q \psi_{q+n}^{c d} \frac{d}{d \psi_{q}^{c d}}+\sum_{q=0}^{n} \phi_{n-q} \phi_{q} \\
\tilde{O}_{n}^{a b}=\sum_{q} q \psi_{q+n}^{a b} \frac{d}{d \phi_{q}}+\sum_{q, c d} \sum_{u=0}^{q-1} \psi_{n+q-u}^{a d} \psi_{u}^{c b} \frac{d}{d \psi_{q}^{c d}}+\sum_{u=0}^{n-1} \phi_{n-u} \psi_{u}^{a b} \\
J_{n}^{a b}=\sum_{m, c} \psi_{m+n}^{c b} \frac{d}{d \psi_{m}^{c a}}+\delta^{a b} \phi_{m} \quad ; \quad \bar{J}_{n}^{a b}=\sum_{m, c} \psi_{n+m}^{a c} \frac{d}{d \psi_{n}^{b c}}+\delta^{a b} \phi_{m} \\
O_{n, u}^{a b}=-\sum_{d} \psi_{n-u}^{a d} J_{u}^{b d}+\sum_{c} \psi_{u}^{c b} \bar{J}_{n-u}^{a c}
\end{gathered}
$$

One introduces the combined operator:

$$
O_{n}^{a b} \equiv \tilde{O}_{n}^{a b}+\sum_{u=0}^{n-1} \psi_{n-u}^{a d} J_{u}^{d b}
$$

in order to have a more symmetric cubic term $\sum_{q, c d} \sum_{u=0}^{n+q-1} \psi_{n+q-u}^{a d} \psi_{u}^{c b} \frac{d}{d \psi_{q}^{c d}}$ 
The Jacobian $J$ is independent of which particular Hamiltonian is chosen; hence it should not depend on $m_{M}$ and $m_{a}$. It follows that it must be annihilated separately by $O_{n}^{0} ; O_{n}^{a b} ; J_{n}^{a b} ; \bar{J}_{m}^{a b}$ and $O_{n, u}^{a b}$. The last is a dependent operator. Notice also that conjugation properties of $\psi$ and $M$ imply that $J_{n}^{a b}=\bar{J}_{n}^{b a}$.

The conjugate operators to $\left\{O_{n}^{0}, O_{n}^{a b}, J_{n}^{a b}, \bar{J}_{n}^{a b}\right\}$ do close a non-linear algebra with a number of characteristic features:

$$
\begin{aligned}
& {\left[O_{n}^{0}, O_{m}^{0}\right]=(m-n) O_{n+m}^{0} \quad \text { (Virasoro algebra of } 1-\text { matrix model) }} \\
& {\left[O_{n}^{0}, \bar{J}_{m}^{a b}\right]=m \bar{J}_{m+n}^{a b}} \\
& {\left[O_{n}^{0}, J_{m}^{a b}\right]=m J_{m+n}^{a b} \quad \text { (Spin } 1 \text { currents) }} \\
& {\left[J_{n}^{a b}, J_{m}^{c d}\right]=-\delta^{b c} J_{n+m}^{a d}+\delta^{a d} J_{n+m}^{c b}} \\
& {\left[\bar{J}_{n}^{a b}, \bar{J}_{m}^{c d}\right]=\delta^{b c} \bar{J}_{n+m}^{a d}-\delta^{a d} \bar{J}_{n+m}^{c b} \quad(\operatorname{sl}(r, \mathbf{C}) \times \operatorname{sl}(r, \mathbf{C}) \text { current algebra })} \\
& {\left[J_{n}^{a b}, \bar{J}_{m}^{c d}\right]=0} \\
& {\left[O_{n}^{0}, O_{m}^{a b}\right]=(m-n) O_{m+n}^{a b}+\sum_{u=0}^{n-1}(n-u)\left(\sum_{g} \psi_{u}^{g b} \bar{J}_{n+m-u}^{a g}+\sum_{h} \psi_{u}^{a h} J_{n+m-u}^{h b}\right)} \\
& {\left[J_{n}^{a b}, O_{m}^{c d}\right]=\delta^{a d} O_{n+m}^{c b}} \\
& -\sum_{u=0}^{n-1}\left\{\psi_{u}^{c b} J_{n+m-u}^{a d}+\delta^{a d} \sum_{f} \psi_{u}^{f b} \bar{J}_{m+n-u}^{c f}\right\} \\
& {\left[\bar{J}_{n}^{a b}, O_{m}^{c d}\right]=\delta^{b c} O_{n+m}^{a d}-\sum_{u=0}^{n-1}\left\{\psi_{u}^{a d} \bar{J}_{n+m-u}^{c b}+\delta^{b c} \sum_{f} \psi_{u}^{a f} J_{n+m-u}^{f d}\right\}} \\
& {\left[O_{n}^{a b}, O_{m}^{c d}\right]=\sum_{v=0}^{m-1} \sum_{u=0}^{m-v-1} \psi_{u}^{c b} \sum_{f} \psi_{n+m-u-v}^{f d} \bar{J}_{v}^{a f}+\sum_{v=0}^{n-1} \sum_{u=0}^{n-v-1} \psi_{v}^{c b} \sum_{h} \psi_{n-u-v}^{a h} J_{n+u}^{h d}} \\
& -\sum_{v=0}^{n-1} \sum_{u=0}^{n-v-1} \psi_{u}^{a d} \sum_{f} \psi_{n+m-u-v}^{f d} \bar{J}_{v}^{c f}-\sum_{v=0}^{m-1} \sum_{u=0}^{m-v-1} \psi_{v}^{a d} \sum_{h} \psi_{m-u-v}^{c h} J_{n+v}^{h b}
\end{aligned}
$$

The closure of this constraint algebra now ensures the existence of the Jacobian from Frobenius-Schur theorem, at least locally.

This algebra realizes a non-Abelian extension of the simplest case $r=1$ studied in [15]. This structure is of an original type. It contains a linear 
current algebra $\operatorname{sl}(r, \mathbf{C}) \times \operatorname{sl}(r, \mathbf{C})$ and a Virasoro algebra. Note that this chiral current algebra contains a current algebra $S U(r)$ generated by $\mathcal{J}_{n}^{a b} \equiv$ $J_{n}^{a b}-\bar{J}_{n}^{a b}$. This current algebra plays a crucial role in the continuum field theory. The non-linear commutators containing the generators $O_{n}^{a b}$ however do not take the form of $W$-algebra commutators; instead of closing over the product of the Virasoro generator with itself they close on the product of bilinear of the fields with the two $\operatorname{sl}(r, \mathbf{C})$ currents.

The hermitian form for the Hamiltonian is obtained from eq. (11); however the matricial nature of the kernel $\Omega_{i j}$ makes it difficult to give an explicit global formula for any value of $r$. Of course $H$ can be computed systematically for each value $r$.

Let us summarize the basic features of the field theory which we have constructed and compare it with previous works. The basic Hamiltonian $H_{1}$ representing the matrix Laplacian, and after reduction the spin Calogero Moser model, consists of three terms:

1) The scalar field self-interaction:

$$
h_{1}=\sum_{n, m} \phi_{n+m} \frac{d^{2}}{d \phi_{n} d \phi_{m}}+\sum_{n} n \sum_{u=0}^{n} \phi_{n-u} \phi_{u} \frac{d}{d \phi_{n}}
$$

representing the well-known one-matrix collective Hamiltonian [2, 7].

2) The pure $S U(r)$ current algebra term:

$$
h_{2}=\sum_{n, m} \sum_{a b, c d} \sum_{u=0}^{n+m} F(n, m, u) \psi_{n+m-u}^{a d} \psi_{u}^{c b} \frac{d^{2}}{d \psi_{n}^{a b} \psi_{m}^{c d}}
$$

which has the same overall form as the $S U(r)$ current-current interaction proposed in [19] as a conformal field version of the pure spin Haldane-Shastry model.

3) The coupling between the bosonic scalar field $\phi$ and the currentalgebra:

$$
h_{3}=\sum_{n, m, a b} n m \psi_{n+m}^{a b} \frac{d^{2}}{d \phi_{n} d \psi_{m}^{a b}}+\sum_{n} \sum_{u=0}^{n}(n-u) \phi_{u} \psi_{n-u}^{a b} \frac{d}{d \psi_{n}^{a b}}
$$

The current-algebra operator coupled to the scalar field is recognized as the energy-momentum tensor $T^{J}(z)$ of the current $\mathcal{J}^{a b}$. Interestingly the same scalar-to-Virasoro-generator coupling occurred in [24, in the context 
of constructing a field theory of the one-matrix model incorporating the topological "discrete" states.

The discrete non-hermitian Hamiltonian (6) can be consistently restricted to the positive-index modes for $\phi$ and $\psi$. Introducing now the conformal field notation :

$$
\begin{aligned}
\Phi(z)=\sum_{n \geq 0} z^{n-1} \phi_{n} \quad ; \quad \Pi(z)=\sum_{n \geq 0} z^{-n} \frac{d}{d \phi_{n}} \\
\alpha(z)=\Phi(z)+\partial_{z} \Pi(z) \quad ; \quad \mathcal{J}^{a b}=\sum_{n \in \mathbf{Z}} \mathcal{J}_{n}^{a b} z^{-n-1}
\end{aligned}
$$

the resulting Hamiltonian reads:

$$
H_{1}=\int d z z^{2}\left(\frac{(\alpha(z))^{3}}{6}+\alpha(z) T^{J}(z)\right)+\int d z \int d y \sum_{a b} \frac{\mathcal{J}^{a b}(z) \mathcal{J}^{b a}(y)}{(z-y)^{2}}
$$

it represents a continuum field theory of a collective boson $\alpha$ interacting with a $S U(r)$ current algebra. It is known from 22, 29 that a more careful definition of the pure current algebra term requires introduction of a cubic term in the continuum theory from considerations of symmetry. These terms could arise, in our present approach, from effects of the non-trivial Jacobian.

\section{The Diagonalization of $H_{1}$}

The Hamiltonian

$$
H_{1}=\sum_{i, j} \operatorname{Tr}\left(e_{i j} U \frac{\partial}{\partial U}\right) \cdot \operatorname{Tr}\left(e_{j i} U \frac{\partial}{\partial U}\right)=\sum_{i, j} \frac{\partial^{2}}{\partial m_{i j} \partial m_{j i}}
$$

is of particular interest since it reduces under the well-known MarsdenWeinstein procedure to the spin Calogero-Moser system. We shall study the spectrum of its restriction to $U(N)$-invariant quantities of the configuration space $\left\{M, x_{i}^{a}, \bar{x}_{j}^{b}\right\}$. Since such quantities are obtained by elimination of the angular degrees of freedom of the configuration space, this spectrum

should be included (not identical, due to the extra permutation symmetry 
of involved in defining the $\phi$-variables) into the spectrum of a spin CalogeroMoser system with complex vector variables. Note that the Hamiltonian derived in 23] from an $N \times \bar{N}$ tensor representation of the Yang-Baxter equation may be interpreted as a restriction to a smaller Hilbert space of this type of Calogero-Moser model.

We will use the nonhermitean collective discrete Hamiltonian (6) derived in the previous section. Its spectrum is the same as the spectrum of its hermitian version, and the eigenstates are obtained a multiplication by the square root of the Jacobian.

The strategy for diagonalizing $H_{1}$ in (6) proceeds from three properties of $H_{1}$ :

Property 1: The number of vector variables $\psi_{n}^{a b}$ is conserved. This is due to the global U(1) symmetry of (6) $\psi_{n}^{a b} \rightarrow \lambda \psi_{n}^{a b}$, itself stemming from the trivial chiral symmetry of $H_{1}$ (which does not act on $x, \bar{x}$ ) under $x_{a} \rightarrow \lambda x_{a}, \bar{x}_{b} \rightarrow \bar{\lambda} \bar{x}_{b}$. It follows that eigenfunctions are separated into selection sectors with a given number $N_{V}$ of vector variables.

Property 2: the total sum of indices $n_{i}$ in $\phi_{n_{i}}, \psi_{n_{j}}^{a b}$ is conserved. This follows from the form of $H_{1}$ as $\operatorname{Tr}\left(U \frac{\partial}{\partial U} \cdot U \frac{\partial}{\partial U}\right)$ which conserves the total number of $U$-type variables. This provides us with a further separation of eigenfunctions into selection sectors with a given number $N_{0}$ of $U$-variables.

Property 3: Define $P\left(N_{0}^{+}, N_{0}^{-}\right)=\left\{\operatorname{Polynomials}\left(\phi_{n}^{0}, \phi_{m}^{a b}\right), \sum_{n, m>0} n_{i}+m_{i}=\right.$ $\left.N_{0}^{+}, \sum_{n, m<0} n_{j}+m_{j}=-N_{0}^{-}\right\}$. The flag vector spaces defined as $F\left(N_{0}, q_{0}\right)=\bigoplus_{q=0}^{q_{0} \geq 0} P\left(N_{0}+q, q\right)$ for $N_{0} \geq 0$ and $\bigoplus_{q=0}^{q_{0} \geq 0} P\left(q,-N_{0}+q\right)$ for $N_{0} \leq 0$, are invariant under $H_{1}$. In particular the polynomials in purely positive or negative index variables $\phi, \psi^{a b}$, are invariant under $H_{1}$.

The proof of Property 3 is obtained by recursion over $q_{0}$. The choice $N_{0} \geq 0$ or $N_{0} \leq 0$ is arbitrary, in fact the eigenvalues and eigenvectors in sectors $N_{0} \leq 0$ are deduced from sectors $N_{0} \geq 0$ by changing $U$ into $U^{-1}$; this keeps the form of $H_{1}$ and changes the indices $n_{i}$; hence eigenfunctions have same eigenvalues with signs of indices $n_{i}$ globally changed.

We choose $N_{0} \geq 0$. When $q_{0}=0$, it is immediate by inspection of (6) that $H_{1}$ generates only positive indices out of positive indices, hence $P\left(N_{0}, 0\right)$ is 
stable. Assume Property 3 to be proved up to $q_{0}$. Consider $P\left(N_{0}+q_{0}+\right.$ $\left.1, q_{0}+1\right)$. From (6), we see that

a) terms linear in derivative pick one index and split it into same-sign pair of indices, hence both $N_{0}^{+}$and $N_{0}^{-}$are conserved.

b) the first two quadratic terms pick pairs $(n, m)$ and join them as $n+m$. If $n, m$ have same sign, this conserves both $N_{0}^{+}$and $N_{0}^{-}$; if not, $N_{0}^{+}$and $N_{0}^{-}$are simultaneously decreased. Hence one ends up in $P\left(N_{0}+q^{\prime}, q^{\prime}\right)$ with $0 \leq q^{\prime}<q_{0}+1$, which is inside the flag vector space.

(c) the third quadratic term picks pairs $(n, m)$ and turns them into pairs $\left(n^{\prime}, m^{\prime}\right)$. If $n, m$ have same sign, $n^{\prime}$ and $m^{\prime}$ keep that same sign and same sum, hence both $N_{0}^{+}$and $N_{0}^{-}$are conserved. If not, say $n \geq 0 \geq m$, $n^{\prime}$ and $m^{\prime}$ stay between $n$ and $m$ with same sum. Hence the positive term (if any) is smaller than $m$; the negative term, if any, is smaller in absolute value than $|m|$; and the sum (if both $n^{\prime}, m^{\prime}$ have same sign) is $n+m$ which is necessarily smaller, in absolute value, than the single index $n$ or $m$ which it replaces in $N_{0}^{+}$or $N_{0}^{-}$. Therefore the third quadratic term sends $P\left(N_{0}+q_{0}, q_{0}+1\right)$ either to itself, or to a lower- $q_{0}$ vector space, stabilizing the flag vector space $F\left(N_{0}, q_{0}\right)$.

The diagonalizing procedure now follows:

1. Fix the values of $N_{0}$ (taken to be positive) and $N_{V}$.

2. Diagonalize $H_{1}$ on the finite vector space $P\left(N_{0}, 0\right)$. This shall be described in more detail later.

3. Diagonalize $H_{1}$ by recursion on the flag spaces $F\left(N_{0}, q_{0}\right) \supset F\left(N_{0}, q_{0}-\right.$ 1) $\supset \cdots \supset F\left(N_{0}, 0\right)=P\left(N_{0}, 0\right)$. The recursion is made possible by the nested structure of $H_{1}$ exhibited in the proof of Prop. 3, namely $H_{1}\left(P\left(N_{0}+q_{0}, q_{0}\right)\right) \subset P\left(N_{0}+q_{0}, q_{0}\right) \cup_{q^{\prime}<q_{0}} P\left(N_{0}+q, q^{\prime}\right)$.

Moreover it follows that one can obtain the spectrum by restricting the matrix of $H_{1}$ to the sole matrix elements inside the space $P\left(N_{0}+q_{0}, q_{0}\right)$. Indeed, diagonalizing this smaller $P\left(N_{0}+q_{0}, q_{0}\right) \rightarrow P\left(N_{0}+q_{0}, q_{0}\right)$ matrix leaves the full $F\left(N_{0}, q_{0}\right) \rightarrow F\left(N_{0}+q_{0}\right)$ matrix as:

$$
\begin{aligned}
H_{1} \mid V_{n} \in P\left(N_{0}+q_{0}, q_{0}\right)> & =\epsilon_{n}\left|V_{n}>+\sum \lambda_{n^{\prime}}\right| V_{n}^{\prime}>\in P\left(N_{0}+q^{\prime}, q^{\prime}\right), q^{\prime}<g_{0} \\
H_{1} \mid V_{n^{\prime}}> & =\epsilon_{n^{\prime}} \mid V_{n^{\prime}}>\text { (recursion hypothesis) }
\end{aligned}
$$


from which the exact eigenstates in $P\left(N_{0}+q_{0}, q_{0}\right) \cup_{q^{\prime}<q_{0}} P\left(N_{0}+q, q\right)$ become:

$$
\begin{aligned}
& H_{1}\left\{\left|V_{n}>+\sum_{V_{n^{\prime}} \in P\left(N_{0}+q^{\prime}, q^{\prime}<q_{0}\right)} \frac{\lambda_{n^{\prime}}}{\epsilon_{n}-\epsilon_{n^{\prime}}}\right| V_{n^{\prime}}>\right\} \\
& =\epsilon_{n}\left\{\left|V_{n}>+\sum \frac{\lambda_{n^{\prime}}}{\epsilon_{n}-\epsilon_{n^{\prime}}}\right| V_{n^{\prime}}>\right\}
\end{aligned}
$$

A potential problem arises if $\epsilon_{n^{\prime}}=\epsilon_{n}$ for some $n^{\prime}$. However this would imply for $H_{1}$ a structure of the form $H_{1} \simeq \epsilon_{n}\left|V_{n}><V_{n}\right|+\epsilon_{n}^{c} \mid V_{n^{\prime}}><$ $V_{n^{\prime}}\left|+\lambda_{n}\right| V_{n^{\prime}}><V_{n} \mid$ which implies that $H_{1}$ is not diagonalizable (by CayleyHamilton theorem) but since $H_{1}$ is conjugate to a hermitian Hamiltonian, this situation cannot occur.

It follows that the computation of all eigenfunctions and eigenvalues of $H_{1}$ can be done by diagonalizing finite-dimensional matrices and we shall now present some specific subsets of eigenfunctions, beginning with the simplest. We shall only consider here the diagonalization in the space $P\left(N_{0}, 0\right)$.

Polynomials in positive-index variables with fixed $N_{V}$ and $N_{0}$ are a finite invariant subset of the full Hilbert space. Two subspaces of $P\left(N_{0}, 0\right)$ have a particularly simple form for $H_{1}$. The first one is the one-matrix, zero-vector subspace $N_{V}=0$ which was investigated fully in [26]. The second one is the subspace of polynomials in $\left\{\psi_{1}^{a b}\right\}$, which is easily seen to be invariant.

The pure one-matrix eigenfunctions are the characters of all representations $R_{n}$ of $S U(N)$, labeled by a Young tableau $Y_{R_{n}}$. The corresponding eigenvalue is given by a simple formula:

$$
\epsilon_{Y}=\frac{1}{2} \sum_{\{\text {lines }} \sum_{\left.n_{1} \geq n_{2} \geq \cdots n_{p}\right\}} n_{k}\left(n_{k}-2 k+1\right)
$$

It turns out that it can also be rewritten as:

$$
\epsilon_{Y}=\frac{1}{2} n_{Y}\left(n_{Y}-1\right) \quad \frac{\chi_{Y}(2-\text { transposition })}{\chi_{Y}(\mathbf{1})}
$$

where $n_{Y}$ is the total number of boxes in $Y ; \chi_{Y}$ is the character function of the permutation group over $n$ elements in the representation of $S_{n}$ labeled by $Y$. In this way a connection is established between representations of $S U(N)$, permutation groups $S_{n}$ and eigenfunctions of $H_{1}$. 2

\footnotetext{
${ }^{2}$ We are indebted to Marc Bellon for pointing out this connection to us.
} 
The connection is even more apparent in the second sector, namely polynomials of degree $n$ in $\psi_{1}^{a b}$. $H_{1}$ reduces to:

$$
H_{1}=\sum_{b_{1}, b_{2}=1}^{n} P_{b_{1} b_{2}} \quad \text { (permutation of } 2 \text { indices of } \mathrm{b}-\text { type) }
$$

Diagonalizing $H_{1}$ is now a simple problem in representation theory of $S_{n}$. First of all, one shows that $H_{1}$ commutes with $S_{n}$.

Indeed $H_{1}$ commutes with all 2-transpositions. Consider $P_{a_{1} a_{2}}$. Contributions to $H_{1} \cdot P_{a_{1} a_{2}}-P_{a_{1} a_{2}} \cdot H_{1}$ only come from the permutations $P_{a_{1}\left(b_{2} \neq a_{2}\right)}$, $P_{b_{1} \neq a_{1}, a_{2}}, P_{a_{1} a_{2}}$ in $H_{1}$. $P_{a_{1} a_{2}}$ commutes with itself, and it is easy to check that

$$
\left[P_{a_{1} a_{2}}, P_{a_{1} b}+P_{a_{2} b}\right]=0
$$

From this and Schur's lemma, it follows that $H_{1}$ is identical to $\lambda_{R} \cdot \mathbf{1}$ on any representation $R$ of $S_{n}$. Moreover the value of $\lambda_{R}$ is immediately given as $\lambda_{R}=\frac{\operatorname{Tr} H_{1}}{\operatorname{Tr} 1}$ and from (29) it follows that, given a representation $R$ with Young tableau $Y_{R}$, one has

$$
e_{Y}=\frac{1}{2} n_{Y}\left(n_{Y}-1\right) \frac{\chi_{Y}(2-\text { transposition })}{\chi_{Y}(\mathbf{1})}
$$

Here $n_{Y}=n$ is the number of boxes in any Young tableau giving a representation of $S_{n}$. We recover the same formula as in the pure one-matrix case, a fact which we shall soon interpret.

The eigenfunctions of (29) are constructed in a canonical way as basis vectors of a given representation $R_{Y}$ with Young tableau $Y$ 27]. Given the lines in $Y$ of length $\left(n_{1} \geq n_{2} \geq \cdots n_{q}\right)$, one first construct all ordered $n$-uples, that is, all the ways of inserting the numbers from 1 to $n$ into the boxes of $Y_{R}$ such that numbers always increase from left to right in a line and from top to bottom in a column. One then defines two operators:

$$
\begin{aligned}
& P=\prod_{\text {lines }}\{\text { symmetrizing operator over indices in line }\} \\
& Q=\prod_{\text {columns }}\{\text { antisymmetrizing operator over indices in columns }\}(32)
\end{aligned}
$$

Finally one acts by P.Q (Young symmetrizer), once, on each of the originally ordered $n$-uples. 
Interpretation of the identity of eigenvalues in these two different sectors comes from considering the original (unreduced) quadratic Hamiltonian $H_{1}$. $H_{1}$ is a scalar Hamiltonian, invariant under the action of unitary matrices $S U(N)$ on hermitian matrices $M_{i j}$. From Schur's lemma it follows that all its eigenvalues depend only on the choice of a particular representation of $S U(N)$ on which it is made to act. In particular this eigenvalue can be determined by having $H_{1}$ act upon an invariant one-dimensional vector space inside this representation, i.e. the character of $R_{Y}$. Hence the eigenvalues (31) determined in the one-matrix model extend to all eigenfunctions of $H_{1}$ identified with explicit matrix elements in a particular representation. In particular the polynomials eigenfunctions of (6) are expectation values between particular vectors $<\bar{x}_{a_{1}} \otimes \cdots \bar{x}_{a_{n}} \mid$ and $\mid x_{b_{n}} \otimes \cdots x_{b_{1}>}$ of $M^{\otimes n}$ symmetrized according to a particular $n$-box Young tableau so as to get an irrep. of $S U(N)$. Note that indeed $H_{1}$ does not act on $x, \bar{x}$ as dynamical variables.

It immediately follows that the study of more complicated eigenstates combining $\phi_{n}^{0}$ and $\psi_{n}^{a b}$ will generate the same spectrum, again with eigenfunctions interpreted as matrix elements - or linear combinations thereof - of a given representation. We have been able to work out all eigenvalues and eigenfunctions up to $N_{0}=4$; the first examples are presented here:

$$
\begin{array}{ll}
N_{0}=1, \quad & N_{V}=1: \psi_{1}^{a b}, \epsilon=0 \\
& N_{V}=0: \phi_{1}, \epsilon=0 \\
N_{0}=2, \quad & N_{V}=0: \text { characters of representation }(\square)^{\otimes 2} \text { of } S U(N) ; \epsilon= \pm 2 \\
& N_{V}=1: \psi_{2}^{a b} \pm \psi_{1}^{a b} \phi_{1} ; \quad \epsilon= \pm 2 \\
& N_{V}=2: \text { permutation representation }, \psi_{1}^{a b} \psi_{1}^{c d} \pm \psi_{1}^{a d} \psi_{1}^{a b} ; \epsilon= \pm 2 \\
N_{0}=3, \quad N_{V}=0: \text { characters of representation }(\square)^{\otimes 3} \text { of } S U(N) ; \epsilon= \pm 6,0 . \\
\quad N_{V}=1:\left\{\psi_{3}^{a b}-\psi_{1}^{a b} \phi_{2}, \psi_{2}^{a b} \phi_{1}^{0}-\psi_{1}^{a b} \phi_{2}\right\}: \epsilon=0 \\
2 \psi_{3}^{a b} \pm 2 \psi_{2}^{a b} \phi_{1} \pm \psi_{1}^{a b} \phi_{2}+\psi_{1}^{a b}\left(\phi_{1}\right)^{2}: \epsilon= \pm 6 \\
\quad N_{V}=2:\left\{\psi_{2}^{a b} \psi_{1}^{c d}-\psi_{1}^{a b} \psi_{2}^{c d} ; \psi_{2}^{a b} \psi_{1}^{c d}-\psi_{1}^{a b} \psi_{1}^{c d} \phi_{1}\right\}: \epsilon=0 \\
\left(\psi_{2}^{a b} \psi_{1}^{c d}+\psi_{1}^{c b} \psi_{2}^{c d}\right) \pm\left(\psi_{1}^{a d} \psi_{2}^{c b}+\psi_{2}^{a d} \psi_{1}^{c b}\right)+\left(\psi_{1}^{a d} \psi_{1}^{c b} \pm \psi_{1}^{a b} \psi_{1}^{c d}\right) \phi_{1}: \epsilon= \pm 6 \\
N_{V}=3: \text { permutation representation }, \epsilon= \pm 6,0 . \\
N_{V}=0: \text { characters of }(\square)^{\otimes 4} ; \quad \epsilon= \pm 12, \pm 4,0 \\
N_{0}=1: \epsilon=0 \text { once, } \pm 4, \text { twice each, } \pm 12 \text { once } \\
N_{V}=2: \text { two types of eigenstates : }
\end{array}
$$


(with antisymmetrized vector index) : $\epsilon=0, \pm 4$ (twice), -12

(with symmetrized vector index) $: \epsilon=0, \pm 4$; (twice),+12

The eigenvalues are defined up to a shift by $2 N_{0} \phi_{0}$, since $\phi_{0}$ is in fact not a variable but a $c$-number which may be given formally any value. We have not given the forms of eigenfunctions for $N_{0}=4$ since they are quite cumbersome and, due to the degeneracy, not canonical anyway.

We may now apply the diagonalizing scheme developed above to obtain eigenfunctions containing both positive and negative indices, but we shall leave this rather technical problem for the moment. An interesting extension of our discussion is now the introduction of interaction terms in the matrix-vector model, leading to external potential terms in the collective hamiltonian. In particular the construction of exactly algebraically solvable theories should be possible on similar schemes to the one in [24]. The relevant algebra is here the non-linear structure constructed in [25].

\section{The Classical Collective Field Theory}

We now discuss a collective description of the classical spin Caloger Moser model, in order to illuminate the basic properties of the theory. We consider here the real vector case. After fixing the momentum map the reduced matrix model is written in terms of the eigenvalues $\lambda_{i}, i=1 \cdots N$ and the vector degrees of freedom as

$$
H_{1}=-\sum_{i=1}^{N} \frac{\partial^{2}}{\partial \lambda_{i}^{2}}+\sum_{i \neq j} \frac{Q_{i j} Q_{j i}}{\left(\lambda_{i}-\lambda_{j}\right)^{2}}
$$

where the charges

$$
Q_{i j}=\sum_{b=1}^{r} a_{i}^{b} a_{j}^{b \dagger}
$$

close a $U(N)$ algebra. This is rewritten as

$$
H_{1}=-\frac{1}{2} \sum_{i=1}^{N} \frac{\partial^{2}}{\partial \lambda_{i}^{2}}+\sum_{i<j} \frac{q_{i}^{a b} q_{j}^{b a}}{\left(\lambda_{i}-\lambda_{j}\right)^{2}}
$$


with the generators

$$
q_{i}^{b c}=a_{i}^{b} a_{i}^{c \dagger}
$$

realizing a $U(r)$ algebra at each site $i$. The classical transition to collective variables is now done with

$$
\begin{aligned}
\phi(x) & =\sum_{i=1}^{N} \delta\left(x-\lambda_{i}\right) \\
\phi \partial \pi & =\sum_{i} p_{i} \delta\left(x-\lambda_{i}\right)
\end{aligned}
$$

describing the scalar density (and current) and

$$
\mathcal{J}^{a b}(x)=\sum_{i=1}^{N} q_{i}^{a b} \delta\left(x-\lambda_{i}\right)
$$

representing a local $U(r)$ current algebra. A simple rewritting of the Hamiltonian leads us to

$$
H_{i}=\int d x \frac{1}{6}\left(\alpha_{+}(x)^{3}-\alpha_{-}(x)^{3}\right)+\frac{1}{2} \int d x \int d y \frac{\mathcal{J}^{a b}(x) \mathcal{J}^{b a}(y)}{(x-y)^{2}}
$$

with $\alpha_{ \pm}=\phi \pm \partial \pi$. The first term is the standard bosonic Hamiltonian for the eigenvalue density [26] while the second term gives the $U(r)$ current algebra Hamiltonian of the form

$$
\sum_{k} k \mathcal{J}^{a b}(k) \mathcal{J}^{b a}(-k)
$$

coinciding with the current-current interaction in [19]. These two terms are apparently decoupled. However one can easily check the Poisson bracket structure:

$$
\begin{aligned}
\left\{\phi \partial \pi(x), \mathcal{J}^{a b}(y)\right\} & =\partial_{x}\left(\mathcal{J}^{a b}(y) \delta(x-y)\right) \\
\left\{\phi(x), \mathcal{J}^{a b}(y)\right\} & =0
\end{aligned}
$$

Consequently the interaction between the momentum density $\pi(x)$ and the current $\mathcal{J}^{a b}(x)$ arises through the Poisson structure. This situation brings to mind the problem which arises when seeking to add a spin-type variable to the relativistic (Ruijsenaars-Schneider [28]) Calogero-Moser system: such an 
addition may not be achieved while keeping the spin variable independent (in the Poisson structure) of the position/momenta variables. The relation might be deeper actually since the commuting quantum spin Hamiltonians constructed from the quantum $R$ matrix structure of the Calogero-Moser system are in fact closer to a Ruijsenaars-Schneider-like system.

A canonical transformation of the fields so as to undo the "off-diagonal" coupling in (43) will then induce an explicit coupling term in the hamiltonian, of the same form as in (23).

The currents themselves obey the algebra

$$
\left\{\mathcal{J}^{a b}(x), \mathcal{J}^{c d}(y)\right\}=\left(\mathcal{J}^{a d}(x) \delta_{b c}-\delta_{a d} \mathcal{J}^{c b}(x)\right) \delta(x-y)
$$

without a central charge.

It is clear that in quantization a central charge will arise. Considering the filling of the ground state near the upper and lower Fermi momenta, we have

$$
\left\{\alpha_{ \pm}(x), \alpha_{ \pm}(y)\right\}= \pm 2 \partial \delta(x-y)
$$

while for $\phi(x)=\frac{1}{2}\left(\alpha_{+}-\alpha_{-}\right)$the anomaly cancels. Similarly we then expect that

$$
\mathcal{J}^{a b}(x)=J^{a b}(x)-\tilde{J}^{a b}(x)
$$

where $J^{a b}(x)$ and $\tilde{J}^{a b}(x)$ obey $U(r)$ Kac-Moody algebras with opposite central charges $k$.

The complex vector case may also be treated in this way. Indeed the generators $F_{i j}$ in (1) also admit an oscillator-type representation corresponding to internal symmetry indices of a vector representation of $U(r) \times U(r)$. Precisely one defines the variables:

$$
A_{i}^{a ; 1,2} \equiv\left(x_{i}^{a},-\bar{p}_{i}^{a}\right) ; A_{i}^{\dagger a ; 1,2} \equiv\left(p_{i}^{a},-\bar{x}_{i}^{a}\right)
$$

and the algebraic structure of the classical theory is the same as in the real vector case, although with different reality properties for the currents.

Let us finally comment on another approach, devised independently in [30]. This work uses a different set of collective variables taken to be the spin version of standard densities. In our approach the $S U(r)$ degrees of freedom are dealt with by current algebra fields, as in [19, 22]. A possible comparison therefore could be made from a detailed study of the spectrum in both representations. 


\section{Acknowledgements}

This work was sponsored by CNRS and CNRS-NSF Exchange Programme AI 06-93 (J.A.); and DOE Grant DE-FG02-91ER-40688, Task A, Brown University. J.A. wishes to thank Brown University Physics Department members for their kind support.

\section{References}

[1] A. Jevicki, B. Sakita, Nucl. Phys. B185 (1981), 89; ibid 165 (1980), 511.

[2] J. Avan, A. Jevicki, Phys. Lett. B266 (1991), 35; ibid 272 (1991), 17; Comm. Math. Phys. 150 (1992), 149.

[3] I. Andric, A. Jevicki, H. Levine, Nucl. Phys. B215 (1983), 307.

[4] J. A. Minahan and A. Polychronakos, Phys. Rev. B50, 4236 (1994)

[5] S. Iso, Nucl.Phys. B443 (1995), 581.

[6] H. Awata, Y. Matsuo, S. Odake, J. Shiraishi, Nucl. Phys. B449 (1995), 347.

[7] A. Jevicki, J. Rodrigues, Nucl. Phys. B421 (1994), 278.

[8] M. Ikehara, N. Ishibashi, H. Kawai, T. Mogami, R. Nakayama, N. Sasakura, Phys. Rev. D50 (1994), 7467.

[9] F. Sugino and T. Yoneya, Univ. Tokyo preprint UT-95-8, hep-th 9510137, (1995)

[10] K. Zarembo, Phys. Lett. B327 (1994), 90.

[11] I. Affleck, Nucl. Phys. B185 (1981), 346.

[12] Chung-I Tan and Zheng Xi-te, Phys. Rev. D26, 2827 (1982).

[13] J. A. Minahan, Int. J. Mod. Phys. A8, 3599 (1993); J. A. Minahan and A. Polychronakos, Phys. Lett. B302, 265 (1993). 
[14] V.A. Kazakov, I. Kostov, Nucl.Phys. B386 (1992), 520; I. Kostov, Phys. Lett. B349 (1995), 284.

[15] T. Mogami, Phys. Lett. B351 (1995), 439.

[16] J. Gibbons, T. Hermsen, Physica 11D (1984), 337; S. Wojciechowski, D. Levi, Phys. Lett. 103A (1984), 11.

[17] A. Gorski, N. Nekrasov, Nucl. Phys. B414 (1994), 213.

[18] B. Sutherland, Phys. Rev. A4 (1971), 2019; A 5 (1972), 1372; B. S. Shastry, Phys. Rev. Lett. 60 (1988), 635; F. D. M. Haldane, Z. N. C. Ha, Phys. Rev. B46 (1992), 9359.

[19] F.D.M. Haldane, Z.N.C. Ha, J.C. Talstra, D. Bernard, V. Pasquier, Phys. Rev Lett. 69 (1992), 2021.

[20] D. Bernard, M. Gaudin, F. D. M. Haldane, V. Pasquier, J. Phys.A (Math. Gen) 26 (1993), 5219.

[21] E. Billey, J. Avan, O. Babelon, Phys. Lett . A188 (1994), 263.

[22] K. Schoutens, Phys. Lett. B331 (1994) 335; D. Bernard, V. Pasquier, D. Serban, Nucl. Phys.B428 (1994), 612.

[23] J. Avan, O. Babelon, E. Billey, hep-th 9505091, to appear in Comm. Math. Phys. (1996).

[24] J. Avan and A. Jevicki, Nucl. Phys. B439 (1995) 67

[25] J.Avan, E. Billey, Phys. Lett. A198 (1995), 183.

[26] A. Jevicki, Nucl. Phys. B376 (1975), 75.

[27] W. Fulton, J. Harris, "Representation theory", Springer Graduate Text in Mathematics, vol. 124, pp 45 sqq. ; edited by Springer Verlag, New York (1991).

[28] S.N.M. Ruijsenaars, H.Schneider, Ann. Phys. 170 (1986), 370.

[29] C. Ahn, S. Nam, SNUTP/95-113 preprint, hep-th 9510242. 
[30] H. Awata, Y. Matsuo, T. Yamamoto, preprint YITP 95-20, hepth 9512065 (1995). 